\title{
激光表面改性技术发展研究
}

\author{
张群莉 ${ }^{1,2,3}$, 王梁 ${ }^{1,2,3}$, 梅雪松 ${ }^{4}$, 姚建华 ${ }^{1,2,3}$ \\ (1. 浙江工业大学激光先进制造研究院, 杭州 310032；2. 浙江工业大学机械工程学院, 杭州 310032；3. 高端激光制造装备 \\ 省部共建协同创新中心，杭州 310032；4. 西安交通大学机械制造系统工程国家重点实验室，西安 710049）
}

\begin{abstract}
摘要: 激光表面改性技术利用高能量密度的激光束对零部件局部易损易耗区域进行快速处理, 获得材料表面所需性能, 进而 大幅延长零部件使用寿命, 已在航空航天、石油化工、能源、交通、冶金等领域获得应用。本文针对激光冲击强化、激光淬 火、激光熔覆 3 种典型的激光表面改性技术，梳理了相应技术特点及国内外应用现状，凝练我国相关技术领域面临的差距与 问题，总结激光表面改性技术的重点发展方向：专用合金材料制备、多能场激光复合表面改性、面向现场的激光再制造、激 光冲击强化控形控性、激光表面改性智能化、激光表面微结构化。从政府引导、国产化创新、产业链完善、质量评价标准体 系、人才培养等方面提出发展建议, 以期为我国激光表面改性技术发展提供参考借鉴。
\end{abstract}

关键词: 激光表面改性; 冲击强化; 淬火; 熔覆; 发展趋势

中图分类号: TG178 文献标识码: A

\section{Development of Laser Surface Modification Technology}

\author{
Zhang Qunli ${ }^{1,2,3}$, Wang Liang ${ }^{1,2,3}$, Mei Xuesong ${ }^{4}$, Yao Jianhua ${ }^{1,2,3}$ \\ (1. Institute of Laser Advanced Manufacturing, Zhejiang University of Technology, Hangzhou 310023, China; \\ 2. College of Mechanical Engineering, Zhejiang University of Technology, Hangzhou 310023, China; \\ 3. Collaborative Innovation Center of High-end Laser Manufacturing Equipment, \\ Hangzhou 310023, China; 4. State Key Laboratory for Manufacturing System \\ Engineering, Xi' an Jiaotong University, Xi'an 710049, China)
}

\begin{abstract}
The laser surface modification technology uses high-energy-density laser beams to rapidly process the local vulnerable and consumable areas of components, which can achieve the desired performances on material surfaces and greatly extend the service life of the components. After decades of development, laser surface modification technology has been widely applied to aerospace, petrochemical, energy, transportation, metallurgy and other fields. Taking three typical laser surface modification technologies-laser shock processing, laser quenching, and laser cladding — as examples, this study introduces the technical characteristics and application status of these technologies in China and abroad and analyzes the existing problems. It also proposes the key research directions, including special alloy material preparation, multi-energy-field composited laser surface modification, on-site laser remanufacturing, laser shock processing with controlled shape and performance, intelligent laser surface modification, and laser surface micro-structure preparation. Some development suggestions are further proposed from the aspects of government guidance, domestic innovation, industrial chain
\end{abstract}

收稿日期 : 2020-03-15; 修回日期 : 2020-05-08

通讯作者：姚建华，浙江工业大学激光先进制造研究院教授，研究方向为激光表面改性技术; E-mail: laser@zjut.edu.cn

资助项目：中国工程院咨询项目“我国激光技术与应用 2035 发展战略研究” (2018-XZ-27)

本刊网址：www.engineering.org.cn/ch/journal/sscae 
improvement, establishment of the quality evaluation standards system, and talent training, in hope to provide references for the development of laser surface modification technologies in China.

Keywords: laser surface modification; laser shock processing; laser quenching; laser cladding; development trend

\section{一、前言}

以航空航天、新能源、轨道交通为代表的高 端装备对使用部件的表面性能要求极高 [1], 感应、 喷涂、喷丸、滚压等常规表面强化手段已难以满足 高性能装备的应用需求 [2]。激光技术在改善金属 材料的表面性能、突破传统改性技术应用约束等方 面潜力突出 [3]，被视为现代工业的 “万能加工工 具” “未来制造系统共同的加工手段”。激光表面改 性技术利用激光的高亮度、高方向性、高单色性和 高相干性等特征，通过改变金属材料表面的组织结 构和化学组成来对材料表面进行改性或合金化, 进 而达到改善材料表面性能的目的。在低成本材料表 面制备低价格、高性能的表面涂层, 对于重要零件 材料及其性能的选择匹配与设计制造具有重要意 义 [4 7]。

作为一种表面工程普适性技术，激光表面改性 技术面向我国制造业转型升级的重大需求，直接服 务于重大装备高端部件的性能提升与国产化制造, 具有广泛的适用范围和良好的应用前景。常规的激 光表面改性技术有: 激光淬火、激光退火、激光 熔凝、激光合金化、激光熔覆、激光冲击强化、激 光上釉、激光表面微结构化等。其中，以激光冲击 强化、激光淬火、激光熔覆最为典型, 在现代工业 生产中发挥着重要作用。激光冲击强化技术具有非 接触、无热影响区、可控性强、强化效果显著的优 点 [3 4]。经激光淬火后的材料, 其表面强度比常 规淬火提高 5\% 20\%，淬火层深度可达 $1 \mathrm{~mm}$ ，因 此激光淬火具有加热速度快、热影响区小、热变形 小和可局部实施的优点 [5]。激光熔覆具有稀释度 小、组织致密、涂层与基体结合好等优点 [6]。

本文系统梳理激光冲击强化、激光淬火、激 光熔覆等典型的激光表面改性技术国内外发展 现状，结合产业应用需求剖析技术发展面临的问 题, 总结领域技术重点发展方向并提出后续发展建 议, 以期为我国激光表面改性技术研究提供参考 借鉴。

\section{二、激光表面改性技术的发展现状}

\section{（一）激光冲击强化技术}

激光冲击强化技术在航空航天、船舶、汽车、 石油化工、核工业和高端模具等装备制造领域获 得了较多应用。据统计 [8], 在航空航天和船舶领 域, $49 \%$ 的燃气浴轮发动机构件损伤是由疲劳失 效引起的, 而叶片、机匣和传动部件是最容易发 生疲劳断裂的零部件（见图 1 和图 2)。激光冲击 强化技术成为改善发动机关键零部件疲劳寿命的 必要手段。

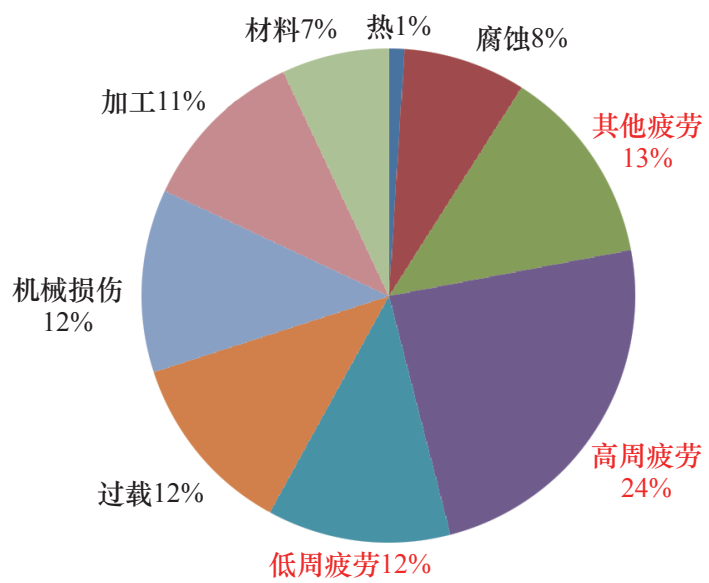

图 1 燃气涡轮发动机失效方式占比分布

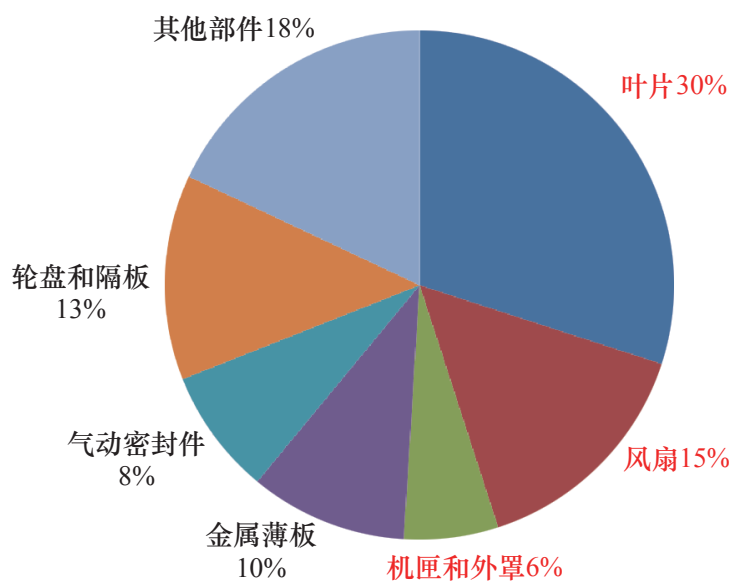

图 2 燃气涡轮发动机部件对高周疲劳的敏感性占比分布 
工业发达国家在早期纷纷开展了激光冲击强化 技术的研究，目前美国仍是激光冲击强化技术发展 最快、应用最成功的国家。美国在 20 世纪 90 年代 后期提出的 “高周疲劳科学与技术研究” 计划, 将 激光冲击强化技术列为先进航空发动机的 76 项关 键技术之一。2012 年，美国金属改性公司（MIC） 研制了移动式激光冲击强化设备以解决现场作业难 题 [1]。美国通用电气公司 (GE) 面向市场需求推 出了用于大型薄壁件加工的激光冲击强化设备, 广 泛应用于发动机叶片 / 叶盘、机翼等结构件的表面 强化。

20 世纪 90 年代, 国内开始了激光冲击强化方 面的技术研究和应用探索。随着激光器技术的快 速发展、相关理论的不断成熟, 我国激光冲击强 化技术的工程应用规模逐渐扩大。2008 年第一条 拥有自主知识产权的激光冲击强化生产线建成投 产，中国成为世界上第二个取得该技术实际应用的 国家 [9]。2011 年，中国科学院沈阳自动化研究所 研制了我国第一台工业应用级整体叶盘激光冲击强 化系统 [10]。2016 年，中国科学院宁波材料技术 与工程研究所在随动式激光冲击强化技术方面取得 突破, 实现了对齿轮、机电腔体、刀具、微细结构 等复杂零件的处理 [11]。江苏大学在高端金属激光 冲击梯度纳米结构形成机制、抗疲劳制造机理、典 型结构冲击强化工艺和应用等方面完成了系列化研 究 [12]。

\section{（二）激光淬火技术}

激光淬火技术在工业发达国家的应用已经普遍 化和成熟化。例如, 诸多汽车制造商都建立了激光 表面淬火生产线, 通过激光表面改性来提高汽车零 部件的耐磨性; 采用激光淬火增加船用柴油机气缸 套内壁的耐磨性，对重载大齿轮进行激光表面淬火 处理来规避常规热处理工艺可能造成的大变形、高 污染问题。

我国激光淬火技术应用起步于 20 世纪 80 年代, 先期在汽车发动机气缸体、缸套等零部件方面开展 应用; 经过多年发展已拓展应用于航空航天、能 源、石化、治金等工业领域，对轴体类、套筒类、 齿轮类、叶片类、模具等零件均取得良好的强化 效果。采用较大光斑的激光束对 $65 \mathrm{Mn}$ 弹簧钢表 面进行淬火, 硬化层深度约为 $0.3 \mathrm{~mm}$, 硬化层硬
度可达基体的 4.2 5.4 倍 [13]。对卷取机卷筒主轴 $40 \mathrm{CrNiMoA}$ 钢表面进行激光淬火, 磨损量仅为基 体的 36.1\% [14]。通过激光淬火对 $35 \mathrm{CrMo}$ 合金结 构钢回转轴截面突变处进行局部强化, 表面残余压 应力是未处理前的 1.9 倍 [15]。针对钢轨在与高速 运行轮对的摩擦配副过程中的损伤问题，华中科技 大学、武汉新瑞达激光工程有限责任公司等单位开 展了钢轨激光淬火强化技术研究, 研制了国际上首 台钢轨激光淬火强化工程车, 获得正式定型并通过 性能考核 [16]。为了解决兆瓦级风力发电机的主轴 轴承在极端环境（重载磨损、高冲击、高盐等）下 的可靠服役问题，浙江工业大学发展了激光深层淬 火技术，将激光淬火层深度提高到 $3.7 \mathrm{~mm}$ ，有效提 高了零部件在复杂环境下的抗疲劳、抗磨损性能(见 图 3 ) [17]。

\section{（三）激光熔覆技术}

激光熔覆技术产生于 20 世纪 70 年代，美国 AVCO 公司率先开展汽车发动机易磨损零部件的表 面强化应用。目前，相关技术在汽车、动力装备、 轨道交通等领域得到广泛应用。日本、美国企业将 激光增材再制造技术商业化，批量修复了军用飞机 发动机的磨损失效零件, 节约经费并提高了修复效 率 [18]。在汽车工业领域 [19], 采用激光熔覆技术 对汽车曲轴进行表面修复, 获得了与曲轴表面结合 紧密且性能良好的熔覆涂层。德国发展了超高速激 光熔覆技术 (EHLA), 可在短时间内完成大面积涂 层的快速制备, 熔覆层厚度在 $0.1 \sim 0.25 \mathrm{~mm}$ 范围可 调, 生产速度比传统激光熔覆提高 100 250 倍, 可 取代电镀、热喷涂、堆焊等传统技术。

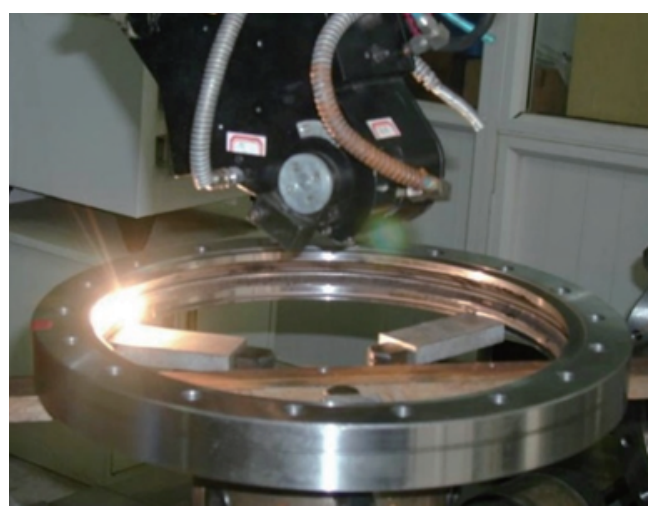

图 3 重载轴承的激光深层淬火 
20 世纪 80 年代以来, 我国激光熔覆技术发展 迅速, 已经从实验室研究扩展到工程实际应用, 如 航空航天、动力装备、机械工业、石油化工、汽车 等行业。机械科学研究总院集团有限公司、哈尔滨 工业大学与国外机构进行联合研发, 实现了超高 速激光熔覆技术的工程应用 [20]。西北工业大学开 展了钛合金零件的激光修复研究, 成功修复了航 空零部件 [21]。浙江工业大学将激光熔覆技术应用 于汽轮机叶片强化和汽轮机转子修复 [22], 对失效 转子进行回收和再制造利用，延长转子使用寿命约 $2 \sim 3$ 倍 (见图 4)。激光熔覆技术大量应用于机械工 业中的轴承、曲轴、模具、螺杆、刀具等易损易耗 零部件。例如, 以激光熔覆技术对天然气净化厂汽 轮机气缸变形位置进行现场修复, 消除了气缸漏气 现象 [23]; 在 45 钢曲轴连杆轴颈上激光熔覆铁基合 金粉末，形成相当于基体 2 3 倍硬度的熔覆层，解 决了曲轴轴颈易出现裂纹和过度磨损的问题 [24]。

\section{三、我国激光表面改性技术面临的问题}

\section{（一）基础理论研究不够深入}

我国激光表面改性技术的研究历程相对较短, 加之重应用、轻机理，使得这一方向的基础知识和 理论研究不够深入透彻, 如基于远平衡态的激光与 材料表面相互作用的材料物理冶金机制、晶粒生长 机制及缺陷形成机制等。

在专用熔覆材料方面，目前多数应用仍然沿用 热喷涂粉末。由于热喷涂与激光束作用是两种完全 不同的物理冶金过程，相关材料实际上不适用于激 光表面强化与再制造, 由此带来的气孔、开裂现象 难以避免。

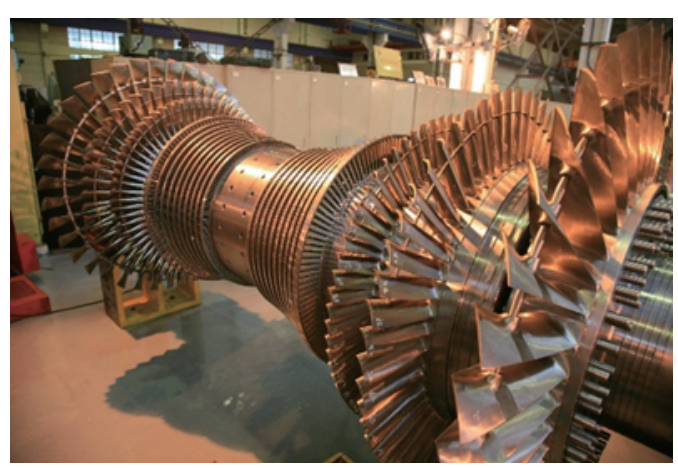

图 4 经激光表面强化后的汽轮机叶片

\section{（二）装备核心部件国产化程度不高}

目前，国内在高质量激光表面改性方面所采用 的激光器多为进口产品, 主要是进口激光器的工作 可靠性和稳定性更好。一方面，进口产品的采购周 期较长, 往往对国内相关技术的发展和研究产生较 大的影响; 另一方面, 激光芯片、激光器、激光头、 控制软件等核心部件国产化水平相对较低，产业发 展受制于人的现象没有消除。

面向未来，激光表面改性将成为主流的制造技 术, 智能化、极端化和高性能化是发展趋势。突破 高效率、高质量激光发生器的国产化研制难题，构 建激光表面改性专用装备集成技术体系，加强批量 生产能力并实现产品化发展, 将是支撑我国制造业 高质量发展的重要挑战之一。

\section{（三）应用市场规范化与技术标准化存在差距}

国内激光表面改性技术的应用仍处于 “小而 散” 的状态，在企业规模、研发投入、产品成熟度、 市场开发、材料研发等多个方面都与国外存在不 小差距。

\section{1. 技术热点}

国外较多关注再制造生产过程中的绿色生产和 质量控制, 相关研究集中在绿色清洗和无损检测技 术方面。国内发表的激光表面熔覆技术工艺研究论 文较多，近期在再制造方向孵化出了一批应用型企 业; 相关研究与应用集中在毛坏损伤修复成形（属 于外形恢复的初级阶段), 而有关修复性能、缺陷 检测等质量保障技术存在较大差距，工艺规范和标 准明显缺失。

\section{2. 制造业应用}

国外激光熔覆技术已经具有了相当的工业体 量, 重点围绕汽车、航空航天、海洋工程装备等高 端零部件的再制造与表面改性实施应用。国内则主 要应用在矿山机械、模具行业、钢铁冶炼、石油化 工、煤炭综采、汽车零件等 “量大面广” 的基础件 修复与耐磨耐蚀性能改善。我国激光表面改性产业 现已初具规模，但在重大、高端装备的应用方面尚 显不足。

3. 产业规模

欧洲多家大型激光表面改性企业不仅完成了在 欧洲的市场布局，还通过代理商模式完成了在我国 主要城市的市场布局。从商业模式看，欧洲企业注 
重生产设备与加工服务相结合, 实际发展也证明这 是推进激光表面熔覆技术产业化的有效形式。相比 之下, 国内激光表面强化企业尚未走出国门来拓宽 海外市场, 在激光制造技术及装备的自主创新发展 方面存在不少短板, 商业运作模式有待优化, 核心 竞争力有待提升。

\section{四、激光表面改性技术的重点发展方向}

\section{（一）激光表面改性专用合金材料制备技术}

激光表面改性（尤其是激光熔覆）的过程是在 高温远平衡凝固条件下进行的, 析出、相变与常规 条件下的演化过程差异甚大。在无法实施后处理的 情况下, 为了获得硬质相的析出, 需要异常高的过 饱和度, 这既是高性能的保障, 又是产生非预期应 力和开裂的主因。研制不易产生缺陷的高性能合金 材料配方是激光表面改性技术实现大范围工业应用 的首要条件。探索基于远平衡态的专用材料设计理 论及科学配比方法, 建立各类专用材料体系, 为激 光表面改性技术的拓展应用提供关键的基础支撑。

\section{（二）多能场激光复合表面改性技术}

高端装备制造业对于关键零部件表面性能的要 求持续提高, 传统的以单一激光作为热源的表面改 性技术面临发展瓶颈。多物理场协同作用的复合改 性技术在对激光表面改性过程中的传热传质行为进 行灵活调控方面显示出了独特优势。相应的重点发 展方向包括: 超音速激光复合沉积、电场 - 磁场协 同的激光表面改性、激光 - 感应复合表面改性、激 光 - 超声振动复合表面改性、激光 - (电) 化学复 合表面改性、多层梯度复合改性等。此外, 深入研 究多物理场协同作用对于激光表面改性非平衡过程 的影响机理, 建立复合场工艺、激光工艺和改性层 性能之间的关系模型。

\section{（三）面向现场的激光再制造技术}

面向企业现场存在的大型机械不易拆卸、运输 困难等问题，研制方便灵活的移动式激光再制造成 套装备。突破高功率、宽光斑、高稳定性的大面积 连续激光再制造工艺技术，拓宽激光再制造工艺窗 口; 通过过程控制缩短再制造时间，提高激光再制
造层的沉积精度来降低表面粗糙度、减少后续加工 量, 实现修复层性能等于或优于原工件。

\section{（四）激光冲击强化控形控性技术}

航空发动机关键部件的激光冲击强化 (LSP), 兼顾 “性能” “形状” 要求较为困难, 这是当前面 临的技术瓶颈问题。为了推进 LSP 技术的普及发展, 可在以下方面开展工作：攻关激光冲击强化控形控 性技术，同时满足航空发动机关键部件的性能、尺 寸精度以及形状表面完整性的技术要求; 设计更高 效率的新型能量耦合装置, 降低激光器的输出要求, 突破无需预铺设吸收层且对边缘效应不敏感的随动 型激光冲击强化技术; 研究新型激光冲击强化工艺 的基础规律，开展管道、狭窄空间内壁的激光冲击 强化处理应用; 研制长寿命、高可靠、智能化激光 冲击强化专用装备, 实现多类工程化应用; 建立系 列工艺规范。

\section{（五）激光表面改性智能化技术}

激光表面改性涉及多学科交叉, 目前国内激光 表面改性智能化控制技术处于起始阶段。重点发展 方向包括: 通过光学、机械、电气、材料、工艺、 制造、控制、信息、网络等学科知识的深度融合, 连接上下游研究机构和企业进行协同攻关; 突破对 温度、材料、尺寸位置、表面质量的智能化控制技 术, 建立激光表面改性专用材料库及工艺数据库; 开展激光表面改性成套设备的集成及系列化工作， 具备智能化生产能力。

\section{（六）激光表面微结构化技术}

应对多功能、多尺度、高效率、高质量、高精 度等产品综合性能的需求，利用短脉冲、超短脉冲 激光制备功能表面微结构, 成为当前制造科学的研 究前沿。脉冲激光织构技术利用激光的高能量峰值 强度和非线性吸收特性, 制备超疏水、超亲水仿生 微结构, 在半导体材料表面制备减反射微结构, 在 金属表面获得减摩微结构。重点研究激光与材料相 互作用的非线性、非平衡和多尺度过程，构建高时 间/空间分辨率、跨尺度的组织结构; 形成闭环反 馈控制的电子动态调控加工新方法、新技术、新装 备, 拓展可重复、高效率的极限微纳制造能力, 匹 
配国家产业应用重大需求。

\section{五、对策建议}

\section{(一) 发挥政策引导作用}

发挥政府机构对激光制造行业发展的政策引导 作用，明晰近、中期的发展战略、重点领域、技术 政策等；鼓励各方联合，集中力量开展技术攻关， 尽快建立自主可控的关键技术体系。作为智力、资 金、技术密集的高新技术产业，激光表面改性技术 的起步门槛较高、投资风险较大、盈利回报时间较 长。建议各级政府出台产业扶持政策，对企业研发 经费予以适当比例的配套，促进建立减少投资风险、 鼓励技术创新、实现企业转型升级的新机制。

\section{（二）加快关键核心器件的创新与国产化}

加快研制具有自主知识产权的激光表面改性成 套装备和关键核心器件，尽快实现激光发生器、送 粉装置、光学系统、激光加工头、运动系统等的国 产化制造，打破国外技术和产品的壁垒。通过技术 创新和设计优化, 确保国产激光表面改性成套装备 的长期稳定和连续运行，以产品质量提高助力我国 制造业高质量发展。

\section{（三）完善产业链配套措施}

支持激光表面改性技术及装备的应用创新和示 范，通过技术进步促进相关产业发展。整合高新技 术企业税收优惠、科技成果转化、科技融资与担保、 孵化器等一系列科技政策和手段, 促进形成涵盖元 器件生产、装备生产、材料制备、软件开发、制造 服务的激光表面改性技术产业链。

\section{（四）建立质量评价标准体系}

成立专业性认定机构, 组织行业专家团队，编 写用于指导激光表面改性的工艺、材料使用、质量 检测、生产安全等方面的标准 (或规范), 建立健 全标准体系。以质量评价标准体系为纽带, 加强 “产学研” 合作和交流，为激光表面改性技术的拓 展应用提供参照和依据。

\section{（五）加强高端人才培养和国际交流合作}

围绕激光表面改性技术对高端技术人才的需
求，为激光表面改性技术的发展提供良好的科研环 境, 培养和打造高水平、梯次合理的科研队伍。发 挥行业学会/协会、高等院校、科研院所及各类相 关社会机构的教育和交流作用，为行业持续发展给 予人才保障。通过国际高端智力引进, 推动我国 激光表面改性技术与装备的创新突破。注重国际 交流与合作，参与国际标准制定以增强产业发展 话语权。

\section{参考文献}

[1] Salimianrizi A, Foroozmehr E, Badrossamay M, et al. Effect of laser shock peening on surface properties and residual stress of Al6061-T6 [J]. Optics and Lasers in Engineering, 2016, 77: 112117.

[2] Manson S S. 金属疲劳损伤 [M]. 陆索, 译. 北京: 国防工业出版 社, 1976.

Manson S S. Metals fatigue damage [M]. Translated by Lu S. Beijing: National Defense Industry Press, 1976.

[3] 喷丸强化技术编写组. 喷丸强化技术 [M]. 北京: 国防工业出版 社, 1973.

Preparation Group of Shot Peening Technology. Shot peening technology [M]. Beijing: National Defense Industry Press, 1973

[4] Kalainathan S, Sathyajith S, Swaroop S. Effect of laser shot peening without coating on the surface properties and corrosion behavior of 316L steel [J]. Optics and Lasers in Engineering, 2012, 50(12): 1740-1745.

[5] 左敦稳. 现代加工技术(第2版) [M]. 北京: 北京航空航天大学出 版社, 2009 .

Zuo D W. Modern processing technology (2nd edition) $[\mathrm{M}]$. Beijing: Beihang University Press, 2009

[6] 徐滨士, 董世运, 王志坚, 等. 激光再制造 [M]. 北京: 国防工业 出版社, 2016.

Xu B S, Dong S Y, Wang Z J, et al. Laser remanufacturing [M]. Beijing: National Defense Industry Press, 2016.

[7] 孙否强, 赵作福, 莫梓睿, 等. 激光技术在材料表面改性方面 的研究进展 [J]. 辽宁工业大学学报(自然科学版), 2018, 38(6): 31-34.

Sun L Q, Zhao Z F, Mo Z R, et al. Research progress of laser technology in surface modification of materials [J]. Journal of Liaoning University of Technology (Natural Science Edition), 2018, 38(6): 31-34.

[8] 张海琼. 航空燃气浴轮发动机叶片疲劳分析 [D]. 哈尔滨: 哈尔 滨工程大学(硕士学位论文), 2014.

Zhang H Q. Fatigue analysis of aero combustion turbine blades [D] Harbin: Harbin Engineering University (Master's thesis), 2014.

[9] 乔红超, 赵吉宾, 陆䒯. 激光诱导冲击波应用技术研究现状 [J]. 表面技术, 2016, 45(1): 1-6, 48.

Qiao H C, Zhao J B, Lu Y. Current status of laser-induced shockwave application technology [J]. Surface Technology, 2006, 45(1): $1-6,48$

[10] 乔红超, 高宇, 赵吉宾, 等. 激光冲击强化技术的研究进展 [J]. 中 国有色金属学报, 2015, 25(7): 1744-1755. 
Qiao H C, Gao Y, Zhao J B, et al. Research process of laser peening technology $[\mathrm{J}]$. The Chinese Journal of Nonferrous Metals, 2015, 25(7): 1744-1755.

[11] 中国科学院宁波材料技术与工程研究所. 新一代激光冲击强化 技术研究获得突破 [J]. 表面工程与再制造, 2017, 17(1): 53.

Ningbo Institute of Material Technology and Engineering, CAS. A new generation of laser shock peening technology get a breakthrough [J]. Surface Engineering \& Remanufacturing, 2017, 17(1): 53.

[12] 鲁金忠. 激光冲击强化铝合金力学性能及微观塑性变形机理研 究 [D]. 镇江: 江苏大学(博士学位论文), 2010.

Lu J Z. Investigation of laser shock processing on the mechanical properties and micro-plastic deformation mechanism of LY2 aluminum alloy [D]. Zhenjiang: Jiangsu University (Doctoral dissertation), 2010 .

[13] 杜成明, 朱锦云, 杨振, 等. $65 \mathrm{Mn}$ 弹簧钢表面激光淬火的显微组 织及性能研究 [J]. 机械工程师, 2020 (3): 52-53.

Du C M, Zhu J Y, Yang Z, et al. Study on laser quenched surface microstructure and properties of $65 \mathrm{Mn}$ spring steel [J]. Mechanical Engineer, 2020 (3): 52-53.

[14] 杨振, 焚湘芳, 邱长军, 等. 激光功率对 $40 \mathrm{CrNiMoA}$ 钢表面淬 火组织和摩擦磨损性能的影响 [J]. 金属热处理, 2020, 45(3): 128-133.

Yang Z, Fan X F, Qiu C J, et al. Effect of laser power on quenched microstructure and friction and wear properties of $40 \mathrm{CrNiMoA}$ steel [J]. Heat Treatment of Metals, 2020, 45(3): 128-133.

[15] 杨秋梅, 周亚军, 毛大恒, 等. 激光淬火对回转轴残余应力影响 的研究 [J]. 热加工工艺, 2020, 49(4): 129-132.

Yang Q M, Zhou Y J, Mao D H, et al. Influence of laser quenching on residual stress of rotary shaft $[\mathrm{J}]$. Hot Working Technology, 2020, 49(4): 129-132.

[16] 杨志翔, 王爱华, 熊大辉, 等. 钢轨表面宽带激光淬火工艺及其 疲劳磨损性能 [J]. 中国机械工程, 2019, 30(3): 253-260.

Yang Z X, Wang A H, Xiong D H, et al. Laser wide-band hardening of steel rails and corresponding fatigue wear property [J] China Mechanical Engineering, 2019, 30(3): 253-260.

[17] 张群莉, 童文华, 陈智君, 等. 光斑尺寸对 $42 \mathrm{CrMo}$ 钢激光深层淬 火硬化层几何特征的影响 [J]. 表面技术, 2020, 49(1): 254-261. Zhang Q L, Tong W H, Chen Z J, et al. Effect of spot size on geometrical characteristics of laser deep quenching hardened layer of 42CrMo steel [J]. Surface Technology, 2020, 49(1): 254-261.

[18] 刘云雷. 镍基高温合金激光再制造零件结合区组织研究与性能 控制 [D]. 南京: 南京航空航天大学(硕士学位论文), 2013.

Liu Y L. Research on microstructure and mechanical property control of bonding zone for laser remanufacturing parts of nickel-based superalloy [D]. Nanjing: Nanjing University of Aeronautics and Astronautics (Master's thesis), 2013.

[19] Koehler H, Partes K, Seefeld T, et al. Laser reconditioning of crankshafts: From lab to application [J]. Physics Procedia, 2010, 5(A): 387-397.

[20] 李俐群, 申发明, 周远东, 等. 超高速激光熔覆与常规激光熔覆 431不锈钢涂层微观组织和耐蚀性的对比 [J]. 中国激光, 2019, 46(10): $1-10$

Li L Q, Shen F M, Zhou Y D, et al. Comparison of microstructure and corrosion resistance of 431 stainless steel coatings prepared by extreme high-speed laser cladding and conventional laser cladding [J]. Chinese Journal of Lasers, 2019, 46(10): 1-10

[21] 薛蕾, 黄一雄, 卢鹏辉, 等. 激光成形修复ZL104合金的组织与性 能研究 [J]. 中国表面工程, 2010, 23(1): 97-100.

Xue L, Huang Y X, Lu P H, et al. Study on microstructure and property of laser forming repaired ZL104 alloy [J]. China Surface Engineering, 2010, 23(1): 97-100.

[22] 赖海鸣, 郭士锐, 姚建华. 基于母材综合跳动特性的转子表面激 光再制造 [J]. 热力透平, 2014, 43(2): 155-158.

Lai H M, Guo S R, Yao J H. Research on characteristics of total indicated runout based on substrate of steam turbine rotor by laser remanufacturing [J]. Thermal Turbine, 2014, 43(2): 155-158.

[23] 肖真. 汽轮机汽缸结合面变形分析及激光熔覆修复 [J]. 石油化 工设备, 2017, 46(3): 51-56.

Xiao Z. Analysis of steam turbine cylinder joint surface deformation and laser cladding repairing [J]. Petro-Chemical Equipment, 2017, 46(3): 51-56.

[24] 封慧, 李剑峰, 孙杰. 曲轴轴颈损伤表面的激光熔覆再制造修复 [J]. 中国激光, 2014, 41(8): 1-6.

Feng H, Li J F, Sun J. Study on remanufacturing repair of damaged crank shaft journal surface by laser cladding $[\mathrm{J}]$. Chinese Journal of Lasers, 2014, 41(8): 1-6. 